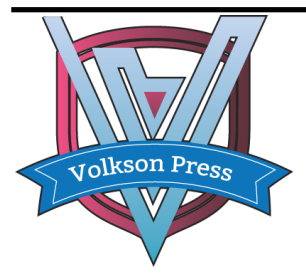

Contents List available at VOLKSON PRESS

Education, Culture and Social Development (ECSD)

DOI : http://doi.org/10.26480/icecsd.01.2018.34.45

Journal Homepage: : https://topicsonsocialdevelop.com/

\title{
A CORPUS-BASED STUDY OF ASEAN COUNTRIES CHINESE SPEAKERS' USE OF CONVERSATIONAL REPAIRS
}

\author{
Xiao Jingling ${ }^{1}$, Zhang Wenguo ${ }^{2 *}$ \\ Department of foreign studies, Guilin University of Technology N0. 12 Jiangan Road, Guilin, China. \\ *Corresponding Author Email: 185576928@qq.com
}

This is an open access article distributed under the Creative Commons Attribution License, which permits unrestricted use, distribution, and reproduction in any medium, provided the original work is properly cited

\section{ARTICLE DETAILS}

\section{Article History:}

Received 12 November 2017

Accepted 12 December 2017

Available online 1 January 2018

\section{ABSTRACT}

The primary purpose of this study was to explore conversational repair types and frequency and repair strategy in ASEAN countries Chinese speakers. The data used in the present study were obtained from the ASEAN countries Chinese speakers' oral Chinese Corpus. This study examined how the repair strategies dealt with communication breakdown in dialogues based on corpus linguistics from a conversational analysis perspective. The results derived from the study were following: Firstly, the author identified 3780 repairs in the study. The preference patterns (SISR and OISR) reflect a widespread but unspoken rule that all participants in a conversation among equals be given a chance to say what they intend to say by themselves.

\section{KEYWORDS}

ASEAN countries Chinese speakers, conversational repair, corpus

\section{INTRODUCTION}

During various kinds of conversations, no matter daily or institutional, we often confront with many kinds of repairs by the speaker or hearer. Repair in this sense is usually termed as an activity a participant conducts to remove troubles in conversation when he or she has difficulty understanding or hearing what another has said or figures what has been said might be wrong, inappropriate [1]. There are copious amounts of repairs in conversation with signals such as pauses, hesitations, false starts as well as rephrases. Repair mechanism is crucial to language acquisition, for it leads to noticing discrepancies between what is said and what is heard, and to a resolution of these discrepancies and although it is not a sufficient condition, it is a necessary condition in language development. Researchers from the seventies of 20th century have contributed a lot to the studies on repair organization in conversation from different perspectives [2]. However, the study of repair and repair strategies used by ASEAN countries Chinese speakers has not received enough attention so far. So, the primary purpose of this paper was to explore conversational repair types and frequency and repair strategies employed by ASEAN countries Chinese speakers based on the corpus linguistics from the conversational analysis perspective to investigate the similarities and differences of conversational repair.

\section{BODY PART}

\subsection{Conversation Analysis}

Studies on practices of conversational repair began with those on the ordinary conversation of native English speakers conducted by a group of scholars using the conceptual and methodological framework of conversation analysis [3]. Historically, conversation analysis began life in the late 1960s and early 1970s. Initially conversation analysis researchers focused on describing the organizational structure of mundane, ordinary conversation between friends and acquaintances either face-to-face or on the telephone.

While conversation analysis studies have focused on native speaker interactions in everyday conversation, in second language acquisition, repair has received attention as a critical factor for nonnative speakers' second language acquisition [4]. Recently, there has been a growing body of conversation analytic work on repair practices in institutional talk and native/non-native conversations.

The methodology of conversation analysis is qualitative in that "conversation analysis attempts to explicate in terms of the conversational practices that speakers orient to by unpacking the structure of either single cases or collections of talk-in-interaction". Conversation analysis practitioners aim to discover the principles with a participant-based perspective [5]. Conversation analysis establishes the perspective not by interviewing research participants, but by examining the details of the "procedural infrastructure of situated action".

\subsection{Definition of Repair}

There are considerable debates on appropriate ways of defining repair and there is no strong consensus yet.

In order to identify ASEAN countries Chinese speakers' conversational repair types and frequencies and repair strategies, the operational definition of a repair for this study has to be clear. This study uses the definition of repair given in some of the most influential studies of repair in ordinary conversation, and classroom settings. In these studies repair is defined as the treatment on the problems of hearing or understanding [6] It includes processes for mutual comprehension such as word search as well as a replacement or correction on hearable errors or mistakes. In the present study the definition and classification of repair organization are based on SJS' theory on repair in the process of identifying and collecting the repairs in the corpus; and it is argued that no systematic analysis of the collected data can be carried out without a reliable system of classification.

\subsection{Corpus-based Approach}

Manually processing research data has long been a tradition since the beginning of research of human language. However, this way is prone to subjectivity and error and it is not always exhaustive. "Corpora are becoming a mainstream", Corpus-based research is also becoming a mainstream [7]. While because of the availability of the means of corpus, computer and software, researchers are now able to conduct researches on various subjects using this burgeoning approach: corpus-based approach.

The present study utilizes the corpus-based approach combining quantitative and qualitative analysis. Biber et al have proposed that corpus-based approach should be.

1) It is empirical, analyzing the actual patterns of use in natural texts;

2) It utilizes a large and principled collection of natural texts, known as a "corpus" as the basis for analysis;

3) It makes extensive use of computers for analysis, using both automatic and interactive techniques; 
4) It depends on both quantitative and qualitative analytical techniques.

\subsection{Research tool}

The main research tool of the present study is Wordsmith Tools Version 4.0 which was designed by Mike Scott and published by the Oxford University. It is composed of six programs : Concord, Wordlist, Keywords, Splitter, Text Converter and Viewer, among which the former three ones are principal programs : Concord can be used to show a collection of the occurrence of a word and it can present co-occurrence information; Wordlist can be used to make a list of words or word clusters and make an index list ; Keywords can be used to find the key words and their location in texts. In the present study Concord and Viewer were used.

\section{DESCRIPTION AND DISCUSSION OF THE CONVERSATIONAL REPAIRS}

In the present study, the author identified 263 conversational repairs. The author also puts all the conversational repairs found in the works into corresponding patterns, whose frequency distribution is shown in the following picture. Table 1 shows the frequency distribution of conversational repair patterns in Thunderstorm.

Table 1: Frequency Distribution of Conversational Repair Patterns in Thunderstorm

\begin{tabular}{|l|l|l|l|l|l|l|l|}
\hline Patterns & SISR & OISR & $\begin{array}{l}\text { SIO } \\
\text { R }\end{array}$ & OIOR & SR & OR & Total \\
\hline $\begin{array}{l}\text { number } \\
\text { s }\end{array}$ & 68 & 106 & 53 & 36 & 174 & 89 & 263 \\
\hline $\begin{array}{l}\text { percent } \\
\text { age }\end{array}$ & $\begin{array}{l}25.9 \\
\%\end{array}$ & $\begin{array}{l}40.4 \\
\%\end{array}$ & $\begin{array}{l}20.1 \\
\%\end{array}$ & $13.6 \%$ & $\begin{array}{l}66.2 \\
\%\end{array}$ & $\begin{array}{l}33.8 \\
\%\end{array}$ & $100 \%$ \\
\hline
\end{tabular}

Table 1 shows that OISR (40.4\%) apparently outnumbers other conversational repair patterns with 106 cases of occurrences and SISR (25.9\%), with 68 cases of occurrences, is also more than SIOR $(20.1 \%)$, with 53 cases of occurrences and OIOR (13.6\%), with 36 cases of occurrences. At the same time Table 3.1 shows that in sharp contrast to the frequency of OR $(33.8 \%)$, SR is $66.2 \%$.

OISR, based on the conclusion of SJS (1977), occurs less frequently than SISR in English conversation. But it is not the case for Chinese conversations according to our corpus. The distribution of different patterns of conversational repairs exhibits a tendency that is different from SJS's observation, which SISR is relatively more favored in real-life conversation. In Chinese drama conversations, OISR is most favored.

\subsection{Explanation for the Results}

As we have ever mentioned, this difference is attributable to the following explanations. Taking the successful communication as the goal, repairs in conversation, in most cases, may rely on the assumptions made by the speaker about how the hearer will recognize what he or she intended to say [8]. If mutual knowledge offers the basis for conversational communication, then each speaker must have the assumption that knowledge he or she takes for granted is in fact shared by the others. However, occasionally the speaker's assumption about the hearer's state of knowledge may be wrong, and the knowledge the speaker takes for granted is actually not shared by the hearer. Repair may possibly occur in such situations.

ASEAN countries Chinese speakers Chinese is a mirror of the society. Chinese culture is a "face culture". So, in conversations, the speakers and hearers unconsciously take face into consideration. As for conversational repair mostly stem from inappropriate forms rather than real errors, the speaker himself is not easy to detect such forms in most cases until the hearer points them out with a quite polite means by issuing other initiation. Then the speaker repairs what he or she has said previously [9]. This can explain why there is a preference for other-initiation over selfinitiation in Chinese drama conversational repairs.

With regard to SJS's viewpoint that self-repair is preferred over otherrepair, we now seek to examine the validity of this argument to conversational repair based on our research results. As indicated in the table, SR, accounting for $66.2 \%$, apparently outnumbers OR, only accounting for $33.8 \%$. Such a distribution suggests that no matter who initiates repair, the speaker of the trouble-source turn will be given the priority of making a self-repair. On this point, our findings about conversational repair in ASEAN countries Chinese speakers' Chinese conversations are generally in conformity with those of SJS's for conversational repair in real-life conversation.
Firstly, the knowledge or background information about the intended entity is a very important factor to determine which side of the participants to perform the repair. It is reasonable to say that the speaker who makes establishment in conversation knows more clearly about the meaning he intends to refer to. The knowledge about the meaning that the speaker takes for granted may or may not be shared by the hearer himself. It is only supposed by the speaker that the hearer has the related knowledge about the intended meaning. Even though the hearer may make inferences or give guesses, the speaker himself always has the priority and the responsibility to make clear his reference for a successful communication

Secondly, other-repair, as holds, may pose a potential face threat in daily conversation between approximate equals, because it is regarded as an interruption of the speaker's current topic and entails a judgment by one speaker about the weakness in the other's speaking ability or knowledge. Other-repair will not be viewed as face-threatening behavior only when it functions as friendly help or serves to further interaction, understanding, and progress toward the common goal. Therefore, the hearer, especially for Chinese, normally will try to behave more politely and avoid committing face-threatening acts by leaving the opportunity for repair to the speaker of the trouble-source turn.

\section{CONCLUSION}

This study explores ASEAN countries Chinese speakers' Chinese repair patterns, frequency and strategies from a relatively stable perspective. One of the central difficulties inherent in the research of repair phenomenon, that is, inherent repair mechanism is not touched in the present study. The mechanism usually involves many factors, such as, learners' language proficiency, their cultural background, gender difference and task requirement and personality and so on. Therefore, further study needs to put such factors into consideration and to investigate the co-relationship among them.

\section{ACKNOWLEDGMENTS}

This study is subsidized by Guangxi Education Department Youth Teacher Project No.KY2016LX104, No. KY2016LX118, and No.2017KY0224.

\section{ABOUT THE AUTHOR}

Xiao Jingling ${ }^{1}, \mathrm{MA}$, assistant professor in Guilin University of Technology, focusing on college English teaching and corpus linguistic studies. Zhang Wenguo ${ }^{2}$, MA, assistant professor in Guilin University of Technology, focusing on formal syntax and theoretical linguistics.

\section{REFERENCE}

[1] Biber, D., Courall, S., Reppen, R. 2000. Corpus Linguistics [M]. Beijing: Foreign Language and Research Press.

[2] Brouwer, C. 2003. Word searches in NNS-NS interaction: Opportunities for language learning [J]. The Modern Language Journal, (87), 534-545.

[3] Buckwalter, P. 2001. Repair sequences in Spanish L2 dyadic discourse: A descriptive study [J]. The Modern Language Journal, (85), 380-397.

[4] Cho, E. 2008. An examination of the use of repair strategies of elementary English as a second language (ESL) students by class types and grade levels [D]. Unpublished doctoral dissertation. Texas A\&M University, College Station.

[5] Duff, P. 2000. Repeat in foreign language classroom interaction [A]. In J.Kelly \& L.S. Verplaetse (Eds.), Second and Foreign Language Learning through Classroom Interaction [C], 109-138.

[6] Hughes, R. 2005. Teaching and Researching Speaking [M]. Beijing: Foreign Language Teaching and Research Press.

[7] Kasper, G. 1985. Repair in foreign language teaching [J]. Studies in Second Language Acquisition, 1 (7), 200-215.

[8] Kurhila, S. 2004. Clients of language learners: being a second language speaker in institutional interaction [A]. In R. Gardner and J. Wagner (Eds.), Second Language Conversations [C]. London: Continuum, 58-74.

[9] Liushu. 2005. Repair in Classroom Interaction: A Conversation Analytic Approach [D]. Unpublished M.A. dissertation, North East Normal University. 